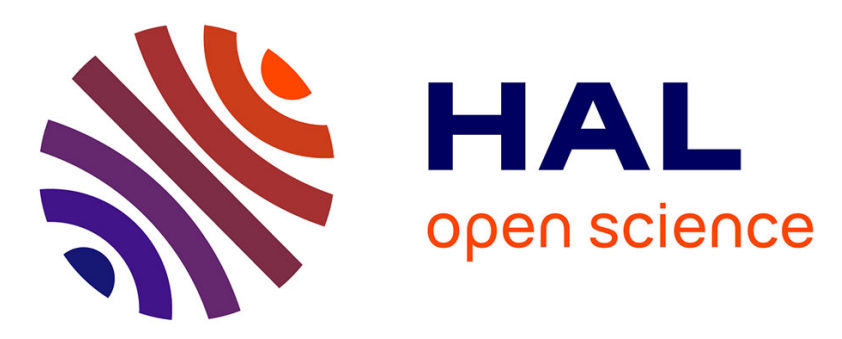

\title{
Equality of the energy and group velocities of bulk acoustic waves in piezoelectric media
}

Vincent Laude, Alexandre Reinhardt, Abdelkrim Khelif

\section{To cite this version:}

Vincent Laude, Alexandre Reinhardt, Abdelkrim Khelif. Equality of the energy and group velocities of bulk acoustic waves in piezoelectric media. IEEE Transactions on Ultrasonics, Ferroelectrics and Frequency Control, 2005, 52, pp.1869. hal-00074907

\section{HAL Id: hal-00074907 https://hal.science/hal-00074907}

Submitted on 24 May 2006

HAL is a multi-disciplinary open access archive for the deposit and dissemination of scientific research documents, whether they are published or not. The documents may come from teaching and research institutions in France or abroad, or from public or private research centers.
L'archive ouverte pluridisciplinaire HAL, est destinée au dépôt et à la diffusion de documents scientifiques de niveau recherche, publiés ou non, émanant des établissements d'enseignement et de recherche français ou étrangers, des laboratoires publics ou privés. 


\title{
Equality of the energy and group velocities of bulk acoustic waves in piezoelectric media
}

\author{
Vincent Laude, Alexandre Reinhardt, and Abdelkrim Khelif
}

\begin{abstract}
The equality of the energy and group velocities of bulk acoustic waves in a lossless piezoelectric medium is demonstrated, with the energy velocity defined from the generalized energy density and the generalized Poynting vector.
\end{abstract}

Index Terms-Bulk acoustic waves; Piezoelectric media; Energy velocity; Group velocity.

\section{INTRODUCTION}

It is well known that the energy and the group velocities of bulk acoustic waves (BAW) in elastic media are equal [1], [2]. The energy velocity, $v_{e}$, is defined as the ratio of the Poynting vector to the energy density, with the help of the Poynting theorem, while the group velocity, $v_{g}$, is defined as the derivative of the phase velocity with respect to the direction of propagation. The group velocity is thus defined on a purely geometrical ground and is by construction normal to the slowness surface. Further properties are the equality of the BAW kinetic and the potential (or strain) energies, and the orthogonality of the slowness vector to the wavefront surface.

In the case of piezoelectric media, we are not aware of a general demonstration of equivalent properties, though generalized expressions have long been known for the energy density and the Poynting vector [1]. Nevertheless, the collinearity of the generalized Poynting vector and the group velocity is routinely used to predict the beam-steering angle, or direction of energy transport. However, Zaitsev and Kuznetsova [3] recently identified a possible discrepancy that can occur if the generalized Poynting vector is used to predict the direction of energy transport in strong piezoelectrics such as lithium niobate. Their argument relies on a dissymmetry of the mechanoelectrical and electromechanical contributions to the generalized Poynting vector. Their work has motivated us to establish the equality of the energy and group velocities of bulk acoustic waves in an arbitrary lossless piezoelectric medium, with the energy velocity obtained from the generalized forms of the Poynting vector and the energy density. This property settles the apparent discrepancy.

\section{BASIC RELATIONS}

We summarize in this section some well known energetic relations for bulk acoustic waves propagating in linear piezoelectric media. Let us first consider a perturbance of an arbitrary linear piezoelectric medium, characterized by the

The authors are with the Institut FEMTO-ST, département LPMO, CNRS UMR 6174, 32 avenue de l'Observatoire, F-25044 Besançn, France (e-mail: vincent.laude@lpmo.edu). strain $S_{i j}$, the stress $T_{i j}$, the electric field strength $E_{k}$ and the electric displacement $D_{k}$. These fields are related by the constitutive relations [1], [4]

$$
\begin{aligned}
T_{i j} & =c_{i j k l} S_{k l}-e_{k i j} E_{k}, \\
D_{j} & =e_{j k l} S_{k l}+\epsilon_{j k} E_{k},
\end{aligned}
$$

where $c_{i j k l}, e_{k i j}$ and $\epsilon_{j k}$ are the elastic, piezoelectric and dielectric tensors, respectively. The repeated summation index convention is used throughout this letter. The time-averaged generalized energy density is given by [1]

$$
W=\frac{1}{2} \operatorname{Re}\left(T_{i j} S_{i j}^{*}+D_{k} E_{k}^{*}\right),
$$

and the time-averaged generalized Poynting vector is

$$
P_{j}=\frac{1}{2} \operatorname{Re}\left(-T_{i j} v_{i}^{*}+\left(\mathbf{E} \times \mathbf{H}^{*}\right)_{j}\right),
$$

where $v_{i}$ is the particle velocity, $H_{i}$ is the magnetic field strength and $*$ denotes complex conjugation.

We then consider specifically a time-harmonic plane wave with angular frequency $\omega$ and slowness vector $s_{j}$. The displacements $u_{i}$ are then of the form

$$
u_{i}(\mathbf{x}, t)=u_{i} \exp \left(\jmath \omega\left(t-s_{j} x_{j}\right)\right)
$$

and similar expressions hold for all fields. We specifically demand that the slowness vector be real-valued, i.e. the medium is lossless and evanescent or inhomogeneous bulk acoustic waves are not considered.

Considering further the quasi-static approximation [1], Maxwell's equations give

$$
\begin{aligned}
E_{i} & =\jmath \omega s_{i} \Phi, \\
(\mathbf{s} \times \mathbf{H})_{j} & =-D_{j}, \\
s_{j} D_{j} & =0,
\end{aligned}
$$

where $\Phi$ is the electric potential. The constitutive relations become

$$
\begin{aligned}
T_{i j} & =-\jmath \omega\left(c_{i j k l} s_{k} u_{l}+e_{k i j} s_{k} \Phi\right), \\
D_{j} & =-\jmath \omega\left(e_{j k l} s_{k} u_{l}-\epsilon_{j k} s_{k} \Phi\right) .
\end{aligned}
$$

The generalized energy density simplifies to

$$
W=\frac{1}{2} \operatorname{Re}\left(\jmath \omega T_{i j} s_{j} u_{i}^{*}\right) .
$$

It can be seen that the electromagnetic part of the energy density vanishes. The generalized Poynting vector becomes

$$
P_{j}=\frac{1}{2} \operatorname{Re}\left(\jmath \omega\left(T_{i j} u_{i}^{*}+D_{j} \Phi^{*}\right)\right),
$$

where $v_{i}=\jmath \omega u_{i}$ has been used in Eq. (4). 


\section{ENERGY VELOCITY}

We now introduce the generalized notation

$$
\bar{c}_{i j k l}=\left\{\begin{array}{ll}
c_{i j k l} & i, l=1,2,3 \\
e_{k i j} & l=4, i=1,2,3 \\
e_{j k l} & i=4, l=1,2,3 \\
-\epsilon_{j k} & i, l=4
\end{array} .\right.
$$

This notation should not be confused with the piezoelectrically stiffened elastic constants [4]. With the generalized displacements, $\bar{u}_{i}$, and stresses, $\bar{T}_{i j}$, defined as

$$
\begin{aligned}
\bar{u}_{i} & =u_{i}, i=1,2,3, \\
\bar{u}_{4} & =\Phi, \\
\bar{T}_{i j} & =T_{i j}, i=1,2,3, \\
\bar{T}_{4 j} & =D_{j},
\end{aligned}
$$

the constitutive relations can be written in a compact way as

$$
\bar{T}_{i j}=-\jmath \omega \bar{c}_{i j k l} s_{k} \bar{u}_{l},
$$

and the Christoffel equation [1] governing the wave dynamics assumes the form

$$
\bar{c}_{i j k l} s_{j} s_{k} \bar{u}_{l}=\rho_{i l} \bar{u}_{l},
$$

with

$$
\rho_{i l}=\rho\left(\begin{array}{cccc}
1 & 0 & 0 & 0 \\
0 & 1 & 0 & 0 \\
0 & 0 & 1 & 0 \\
0 & 0 & 0 & 0
\end{array}\right) .
$$

Contracting the Christoffel Eq. (19) with $\bar{u}_{i}^{*}$ yields the relation

$$
\bar{c}_{i j k l} s_{j} s_{k} \bar{u}_{l} \bar{u}_{i}^{*}=\rho_{i l} \bar{u}_{l} \bar{u}_{i}^{*}=\rho u_{i}^{*} u_{i} .
$$

Inserting Eq. (9) into Eq. (11) we obtain

$$
W=\frac{\omega^{2}}{2} \operatorname{Re}\left(c_{i j k l} s_{j} s_{k} u_{l} u_{i}^{*}+e_{k i j} s_{j} s_{k} \Phi u_{i}^{*}\right),
$$

or by inspection

$$
W=\frac{\omega^{2}}{2} \operatorname{Re}\left(\bar{c}_{i j k l} s_{j} s_{k} \bar{u}_{l} \bar{u}_{i}^{*}\right) .
$$

Using Eq. (21), we observe that this quantity is real and thus

$$
W=\frac{\omega^{2}}{2} \bar{c}_{i j k l} s_{j} s_{k} \bar{u}_{l} \bar{u}_{i}^{*}=\frac{\omega^{2}}{2} \rho u_{i}^{*} u_{i} .
$$

This relation proves the equality of potential and kinetic energies for bulk acoustic waves as in the case of elastic media [2].

The expression for the Poynting vector can similarly be transformed by inserting Eqs. (9-10) into Eq. (12), yielding

$P_{j}=\frac{\omega^{2}}{2} \operatorname{Re}\left(c_{i j k l} s_{k} u_{l} u_{i}^{*}+e_{k i j} s_{k} \Phi u_{i}^{*}+e_{j k l} s_{k} u_{l} \Phi^{*}-\epsilon_{j k} s_{k} \Phi \Phi^{*}\right)$,

or simply

$$
P_{j}=\frac{\omega^{2}}{2} \operatorname{Re}\left(\bar{c}_{i j k l} s_{k} \bar{u}_{l} \bar{u}_{i}^{*}\right) .
$$

Defining the energy velocity as for bulk acoustic waves in elastic media by the ratio of the Poynting vector to the energy density, we obtain at once

$$
\left(V_{e}\right)_{j}=\frac{P_{j}}{W}=\frac{\operatorname{Re}\left(\bar{c}_{i j k l} s_{k} \bar{u}_{l} \bar{u}_{i}^{*}\right)}{\rho u_{i}^{*} u_{i}} .
$$

Furthermore, contracting this expression with $s_{j}$, the following useful relation is obtained

$$
s_{j}\left(V_{e}\right)_{j}=1
$$

as in the case of elastic media [1], [2].

\section{GROUP VELOCITY}

The components of the slowness vector can be written $s_{i}=$ $s n_{i}$ where the $n_{i}$ are the components of a unit vector, i.e. $n_{i} n_{i}=1$. With this notation, the Christoffel equation (19) becomes a generalized eigenvalue equation for the square of the phase velocity, $V=s^{-1}$,

$$
\bar{c}_{i j k l} n_{j} n_{k} \bar{u}_{l}=V^{2} \rho_{i l} \bar{u}_{l} .
$$

The group velocity is defined as [2]

$$
\left(V_{g}\right)_{j}=\frac{\partial V}{\partial n_{j}}
$$

which implies that the group velocity vector is normal to the slowness surface by construction. The group velocity vector can be obtained by differentiating with respect to $n_{j}$ the identity formed by contracting Eq. (29) with $\bar{u}_{i}^{*}$, or

$$
\bar{c}_{i j k l} n_{j} n_{k} \bar{u}_{l} \bar{u}_{i}^{*}=V^{2} \rho_{i l} \bar{u}_{l} \bar{u}_{i}^{*}
$$

which is merely a restatement of Eq. (21). In this equation, $\bar{u}_{l}$ is a function of the unit vector $\mathbf{n}$ since it is the eigenvector associated with the eigenvalue $V^{2}$ of the Christoffel equation (29) for a fixed propagation direction. The differentiation of Eq. (31) with respect to $n_{j}$ results in

$$
\begin{gathered}
2 \bar{c}_{i j k l} n_{k} \bar{u}_{l} \bar{u}_{i}^{*}+\bar{c}_{i \beta k l} n_{\beta} n_{k} \frac{\partial \bar{u}_{l}}{\partial n_{j}} \bar{u}_{i}^{*}+\bar{c}_{i \beta k l} n_{\beta} n_{k} \bar{u}_{l} \frac{\partial \bar{u}_{i}^{*}}{\partial n_{j}} \\
=2 V\left(V_{g}\right)_{j} \rho_{i l} \bar{u}_{l} \bar{u}_{i}^{*}+V^{2} \rho_{i l} \frac{\partial \bar{u}_{l}}{\partial n_{j}} \bar{u}_{i}^{*}+V^{2} \rho_{i l} \bar{u}_{l} \frac{\partial \bar{u}_{i}^{*}}{\partial n_{j}} .
\end{gathered}
$$

This equation can be simplified by considering two different contractions of the Christoffel equation (29). First, the contraction by $\frac{\partial \bar{u}_{i}^{*}}{\partial n_{j}}$ yields

$$
\bar{c}_{i \beta k l} n_{\beta} n_{k} \bar{u}_{l} \frac{\partial \bar{u}_{i}^{*}}{\partial n_{j}}=V^{2} \rho_{i l} \bar{u}_{l} \frac{\partial \bar{u}_{i}^{*}}{\partial n_{j}} .
$$

Second, complex conjugation of Eq. (29) and subsequent contraction by $\frac{\partial \bar{u}_{i}}{\partial n_{j}}$ results in

$$
\bar{c}_{i \beta k l} n_{\beta} n_{k} \bar{u}_{l}^{*} \frac{\partial \bar{u}_{i}}{\partial n_{j}}=V^{2} \rho_{i l} \bar{u}_{l}^{*} \frac{\partial \bar{u}_{i}}{\partial n_{j}} .
$$

Permutation of indices $i$ and $l$, and $\beta$ and $k$, respectively, and consideration of the symmetries $\bar{c}_{i \beta k l}=\bar{c}_{l k \beta i}$ and $\rho_{i l}=\rho_{l i}$ lead to

$$
\bar{c}_{i \beta k l} n_{\beta} n_{k} \bar{u}_{i}^{*} \frac{\partial \bar{u}_{l}}{\partial n_{j}}=V^{2} \rho_{i l} \bar{u}_{i}^{*} \frac{\partial \bar{u}_{l}}{\partial n_{j}} .
$$

Eventually, Eq. (32) simplifies to

$$
\left(V_{g}\right)_{j}=\frac{\bar{c}_{i j k l} n_{k} \bar{u}_{l} \bar{u}_{i}^{*}}{V \rho u_{i} u_{i}^{*}}=\frac{\bar{c}_{i j k l} s_{k} \bar{u}_{l} \bar{u}_{i}^{*}}{\rho u_{i} u_{i}^{*}} .
$$

This is identical with the expression (27) for the energy velocity and incidentally shows that the $R e($.$) operators in$ expressions (26) and (27) can be dropped. We have thus shown the equality of energy and group velocities for bulk acoustic waves in a lossless piezoelectric medium. 


\section{CONCLUSION}

We have demonstrated that the energy and group velocities for bulk acoustic waves are equal in a lossless piezoelectric medium. Our derivation closely parallels and generalizes for piezoelectric media the derivation of Ref. [2], that was limited to purely elastic media. The possible discrepancy pointed out in Ref. [3], that the mechanoelectrical and electromechanical power flows calculated from the generalized form of the Poynting vector for piezoelectric media do not compensate each other, has then no significance for the estimation of the power flow direction. This observation is moreover supported by the experimental results of Havlice et al. [5], who demonstrated that the generalized Poynting vector indeed predicts the correct beam-steering angle of longitudinal waves along the $y$ axis of lithium niobate. We finally observe that attempting to separate the generalized energy density or the generalized Poynting vector into their purely electrical, purely mechanical, mechanoelectrical and electromechanical parts can be confusing, since the wave displacements are obtained as the solution of an eigenvalue problem involving the mixed elastic, electrical and piezoelectric properties of the medium.

\section{REFERENCES}

[1] B. A. Auld, Acoustic Fields and Waves in Solids. New-York: Wiley, 1973, vol. 1 .

[2] D. Royer and E. Dieulesaint, Elastic waves in solids, vol. 1. New York: Wiley, 1999.

[3] B. D. Zaitsev and I. E. Kuznetsova, "The energy density and power flow of acoustic waves propagating in piezoelectric materials," IEEE Trans. Ultrason., Ferroelec., Freq. Control, vol. 50, no. 12, pp. 1762-1765, 2003.

[4] "IEEE standard on piezoelectricity 176-1987," IEEE Trans. Ultrason., Ferroelec., Freq. Control, vol. 43, no. 5, p. 717, 1996.

[5] J. F. Havlice, W. L. Bond, and L. B. Wigton, "Elastic Poynting vector in a piezoelectric medium," IEEE Trans. Sonics and Ultrason., vol. SU-17, no. 4 , pp. 246-249, 1970. 